\title{
Lateral transport in disordered layered media
}

\author{
Zhao-Qing Zhang and Kin-Chit O \\ Department of Physics, Hong Kong University of Science and Technology, Clear Water Bay, Kowloon, Hong Kong
}

(Received 16 January 1996; revised manuscript received 8 March 1996)

\begin{abstract}
We study the physics of the channel-hopping mechanism in disordered layered media in the anisotropic regime using an analytical approach. A classical rate equation is formulated to describe the lateral transport. It is found that the asymptotic lateral transport behaviors can be obtained from the behaviors of the eigenvector of $M^{\dagger} M$ that corresponds to the localization length of the system, where $M$ is a transfer matrix. It is also found that the role played by evanescent waves is to increase the equilibration length beyond all channel localization lengths and, therefore, dictate a one-dimensional-like behavior. [S0163-1829(96)51618-8]
\end{abstract}

Recent studies on wave propagation in disordered layered media have revealed many interesting transport behaviors not seen in other disordered systems. ${ }^{1,2}$ In the tight-binding representation, we use the following Hamiltonian to describe a disordered layered medium:

$$
H=\sum_{\alpha} \varepsilon_{\alpha} a_{\alpha}^{\dagger} a_{\alpha}+t \sum_{[\alpha, \beta]} a_{\alpha}^{\dagger} a_{\beta}
$$

where $\alpha, \beta$ are site indices, and $[\alpha, \beta]$ indicates $\alpha$ and $\beta$ are nearest neighbors. We set $t=1$ as the energy scale. If we choose the $z$ axis as the layering direction, i.e., the direction perpendicular to the layers, the site energy $\varepsilon_{\alpha}$ consists of two parts,

$$
\varepsilon_{\alpha}=\eta_{z}+\delta_{\alpha}
$$

$\eta_{z}$ describes the layer energy and is a constant for all sites lying in a given layer plane at fixed $z$, but varies randomly as $z$ varies with a flat distribution of width $W_{1} . \delta_{\alpha}$ is a random number that varies independently from site to site with a flat distribution $W . W_{1}$ and $W$ measure the strengths of layer randomness and isotropic randomness, respectively. In the presence of $W_{1}$, all states are localized along the $z$ direction. These localized wave functions act as overlapped waveguides (or channels) for the propagation of waves along the layer planes. The scatterings caused by the isotropic randomness $W$ introduce hoppings among these channels and facilitate the transport in the $z$ direction. The study of localization phase diagrams in three-dimensions (3D) has indicated that the system behaves $1 \mathrm{D}$-like if $W_{1} / W \geqslant 4 .{ }^{1}$ These results are in sharp contrast to the results of the anisotropic hopping model, where localization transitions were found to occur at any anisotropy. ${ }^{3}$ The physical systems relevant to the Hamiltonian in Eqs. (1) and (2) are the Earth's subsurface and random superlattices with inhomogeneities.

In 2D, the recent numerical study of this Hamiltonian in the anisotropic regime, i.e., $W_{1}=15$ and $W=1.5$, has revealed new transport behaviors caused by channel hoppings. For instance, it has been found, via evanescent waves, that a dominant channel will form when the sample size is larger than an equilibration length and the relative occupation probability among various propagating channels reaches a fixed distribution which is independent of the initial injection channel. ${ }^{2}$ In order to understand the physics of this hopping mechanism, we have studied this problem using an analytic approach.

Let us consider a 2D system of $N_{0}$ layers in the $x-z$ plane with lattice constant $a=1$. For any particular layer configuration, i.e., a given set of $\left\{\eta_{z}\right\}$ for $z=1,2, \ldots, N_{0}$, one can solve the $1 \mathrm{D}$ eigenvalue problem along the $z$ direction and obtain a set of $N_{0}$ eigenfunctions $\varphi_{n}(z)$ and eigenvalues $E_{n}$. The wave energy can be written as $E=E_{n}+2 \cos k_{n}$, where $k_{n}$ is a wave vector of the $n$th channel for the propagation along the $x$ direction. When $\left|E-E_{n}\right|>2, k_{n}$ is imaginary and the channel is denoted "evanescent." When $\left|E-E_{n}\right|<2$ and $k_{n}$ is real, the channel is denoted "propagating." We connect the disordered sample of length $L$, where $W \neq 0$, to two pure leads on both sides of the sample. These leads have the same layer configuration but $W=0$. If there are $N$ propagating channels in the system, the $N \times N$ matrix $t_{n m}$, which denotes the transmission amplitude with incoming wave at the $m$ th channel and outgoing wave at the $n$th channel, can be calculated numerically using the recursive Green's function method. ${ }^{4}$

In order to analyze the structure of $t(L)$ in the large $L$ limit, we make use of a relation between $t^{\dagger}(L) t(L)$ and $M^{\dagger}(L) M(L),{ }^{5}$ i.e.,

$$
\begin{aligned}
& \left(\begin{array}{cc}
t^{\dagger}(L) t(L) & 0 \\
0 & t^{\prime}(L) t^{\prime \dagger}(L)
\end{array}\right) \\
& \quad=\frac{4}{M^{\dagger}(L) M(L)+\left[M^{\dagger}(L) M(L)\right]^{-1}+2}
\end{aligned}
$$

where $M(L)$ is a transfer matrix relating the amplitudes of $N$ left-going channels and $N$ right-going channels on the right lead to those on the left lead. $t^{\prime}$ is the transmission matrix measured in the reverse direction of $t$. Since the order of $M$ is $2 N_{0}$, we have to include also the evanescent channels in $t$ and $t^{\prime}$ to make $N=N_{0}$ in Eq. (3). The element $t_{n, m}$ is nonvanishing only when both channels $n$ and $m$ are propagating. It has been proven by Oseledec ${ }^{6}$ that in the large $L$ limit the Hermitian matrix $M^{\dagger}(L) M(L)$ converges to an asymptotic matrix $M^{\dagger} M$, which has a fixed orthonormal set of eigenvectors, i.e., $f_{i}(n)$ with $i, n=1,2, \ldots, 2 N$, where $n$ denotes the coordinate in channel space. The corresponding eigenvalues have the form $\exp \left(2 \lambda_{i} L\right)$. The functions $f_{i}(n)$ depend on the configuration of isotropic randomness, while the self- 
averaged Lyapunov exponents $\lambda_{i}$ do not. Since $M$ is symplectic, the exponents come in pairs, say, $\lambda_{i}$ and $\lambda_{2 N-i+1}$ $=-\lambda_{i}$ and the eigenfunctions of each pair are related by ${ }^{5}$

$$
f_{i}(n)=\left\{\begin{array}{l}
f_{2 N-i+1}(n), \quad \text { if } \quad 1 \leqslant n \leqslant N \\
-f_{2 N-i+1}(n), \text { if } \quad N+1 \leqslant n \leqslant 2 N .
\end{array}\right.
$$

It is easy to show from Eqs. (3) and (4) that $g_{i}(n)$ $=\sqrt{2} f_{i}(n) \quad(i, n=1,2, \ldots, N)$ and $g_{i}(n)=\sqrt{2} f_{i}(n) \quad(i, n=N$ $+1, N+2, \ldots, 2 N)$ form, respectively, two sets of orthonormalized eigenfunctions of $t^{\dagger} t$ and $t^{\prime} t^{\prime \dagger}$ in the asymptotic regime with identical spectra, i.e., $s_{i}=2 / \cosh \left(2 \lambda_{i} L\right)$, say, 0 $<\lambda_{1}<\lambda_{2} \cdots<\lambda_{N}$ and $s_{2 N-i+1}=s_{i}$. We can write $t^{\dagger} t$ $=S R^{-1} R S$, where $S$ is a contracting Hermitian matrix and is diagonal in the representation of $g_{i}(n)$ with the eigenvalues $\sqrt{s_{i}(L)} . R$ is a unitary matrix. ${ }^{7}$ The matrix elements of $t$ can now be written as

$t_{n m}(L)=\sum_{i=1}^{N} \sqrt{s_{i}(L)} a_{i, n}(L) g_{i}^{*}(m) ; n, m=1,2, \ldots, N$

with

$$
a_{i, n}(L)=\sum_{\ell=1}^{N} R_{n, \ell}(L) g_{i}(\ell) .
$$

Since the off-diagonal elements of $R(L)$ describe the transitions among different channels as a function of sample size $L$ arising from isotropic scatterings, $R(L)$ can be considered as a stochastic process in $L$. In the asymptotic regime, we need only to keep the $i=1$ term in Eq. (5), and $t_{n m}(L)$ becomes

$$
t_{n m}(L) \cong 2 \exp \left(-\lambda_{1} L\right) a_{1, n}(L) g_{1}^{*}(m) .
$$

Thus, $1 / \lambda_{1}$ is the localization length. We can assume $g_{1}(m)$ to be zero for evanescent channel $m$ and limit our discussions below to propagating channels only. From Eq. (7a), we find

$$
t_{n m}(L) / t_{n}(L) \cong g_{1}^{*}(m) / g_{1}^{*}(\ell) .
$$

The equilibration length can be defined as the length required to reach the asymptotic ratios of Eq. (7b). For any incoming channel $m$, the relative occupation probability in the $n$th outgoing channel becomes ${ }^{3}$

$$
\theta_{n}(L)=\frac{\left|t_{n m}(L)\right|^{2}}{\sum_{n=1}^{N}\left|t_{n m}(L)\right|^{2}} \cong\left|a_{1, n}(L)\right|^{2},
$$

where we have used the fact that $R$ is unitary so that the norm of the vector $a_{1, n}$ is unity at any $L$. In the asymptotic regime, the statistics of $\theta_{n}(L)$ are stationary. Therefore, the relative channel occupation distribution is obtained by taking the average of $\theta_{n}(L)$ in $L$. However, in the anisotropic regime where $W_{1} \gg W, R$ is dominated by the diagonal terms. If we approximate the average of $\left\langle R_{i k}(L) R_{i /}^{*}(L)\right\rangle_{L}$ in $L$ by $\delta_{i, k} \delta_{i, \ell}$, we find $\left\langle\theta_{n}\right\rangle_{L} \cong\left|g_{1}(n)\right|^{2}$. Together with Eq. (7b), we find that the asymptotic lateral transport behaviors can be obtained from the behavior of the eigenvector of $t^{\dagger} t$ (or
$M^{\dagger} M$ ) that corresponds to the longest length scale, i.e., the localization length, and is independent of the incoming channels.

The structure of $t_{n m}$ in Eq. (5) also enables us to relate the Lyapunov exponents to the exponential decay of the determinants of various submatrices. It can be shown that for any $M \times M$ submatrix $t^{(M)}$ of $t_{n m}$, the determinant of $t^{(M)}$ has the following asymptotic expression:

$$
\operatorname{det}\left[t^{(M)}(L)\right] \cong A(L) 2^{M} \exp \left\{-\sum_{i=1}^{M} \lambda_{i} L\right\},
$$

where the factor $A(L)$, albeit fluctuating in $L$, does not decay or grow. Thus, Eq. (9) enables us to calculate all the Lyapunov exponents from the decay length of $\operatorname{det}\left[t^{(M)}\right]$. Numerically, we have performed the recursive Green's function calculations for the cases of $N_{0}=2,3,4$, and 6 with periodic boundary conditions along the layer direction $z$. In the case where $W_{1}<2$ and all the channels are propagating, it is found that the equilibration length is on the order of the second longest length scale, i.e., $1 / \lambda_{2}$, and $\left|g_{1}(n)\right|^{2}$ is on the order of $1 / N_{0}$ for all channels. The Lyapunov exponents obtained from Eq. (9) agree well with the results calculated by the standard orthonormalized transfer matrix technique. ${ }^{6,8}$ They are all proportional to $W^{2}$ in the small $W$ limit. This is the typical 1D behavior. Unlike the transfer matrix, the transmission matrix $t_{n m}$ describes physical quantities. In the case of classical waves, Eq. (9) may provide a way to measure the Lyapunov exponents. The transport properties and Lyapunov exponents for elastic waves in randomly layered media have been studied in detail. ${ }^{9}$

In the case of large $W_{1}$, i.e., $W_{1} \gg 2$, there are many evanescent waves. The propagating channels become spatially separated. $^{2}$ In order to consider the role played by the evanescent waves in the lateral transport, instead of having a large value of $W_{1}$, we introduce a large layer energy $W_{b}$ on alternate layers to describe evanescent waves so that we can keep the layer randomness in the propagating layers at $W_{1}$ $<2$. The existence of large $W_{b}$ introduces an effective hopping matrix on the order of $1 / W_{b}$ between two adjacent propagating layers. In principle, we should also allow $W_{b}$ to vary. However, the nature of the transport would not be altered in any qualitative way as long as $W_{1} \gg 1 / W_{b}$ so that the channel wavefunctions are strongly localized. In the following, we present a typical example to illustrate the lateral transport in this regime. We consider here the simplest case of four layers. For an arbitrarily chosen layer configuration of $\eta_{1}=0, \eta_{2}=W_{b}, \eta_{3}=1.6$, and $\eta_{4}=W_{b}$, and $E=0$, there are only two propagating channels for large $W_{b}$. For $W_{b}$ $=1000$, the channel energies are $E_{1}=-0.002$ and $E_{2}$ $=1.598$. For $W=1$, the logarithm of the ratios of $\left|t_{11}(L) / t_{12}(L)\right|^{2}$ and $\left|t_{21}(L) / t_{22}(L)\right|^{2}$ are plotted in Figs. 1(a) and $1(\mathrm{~b})$, respectively, as functions of $L$ for a particular configuration of isotropic randomness. From these curves, it is found that the equilibration length $\xi_{\text {eq }}$ is roughly 650 . Beyond this, we find $\left|g_{1}(1) / g_{1}(2)\right|^{2} \approx 3000$. This shows a dominant concentration of probability in channel one, which has channel energy close to zero. In Fig. 1 (curve C), we also plot the decay of $F_{2}(L)=\left|\operatorname{det}\left[t^{(2)}\right]\right| /\left|t_{11} t_{22}\right|$. It is seen that $F_{2}(L)$ does not decay until $L$ reaches $\xi_{\text {eq }}$. By measuring the decay length of $F_{2}(L)$ after $\xi_{\text {eq }}$, we obtain $\lambda_{2}-\lambda_{1}$. As a 


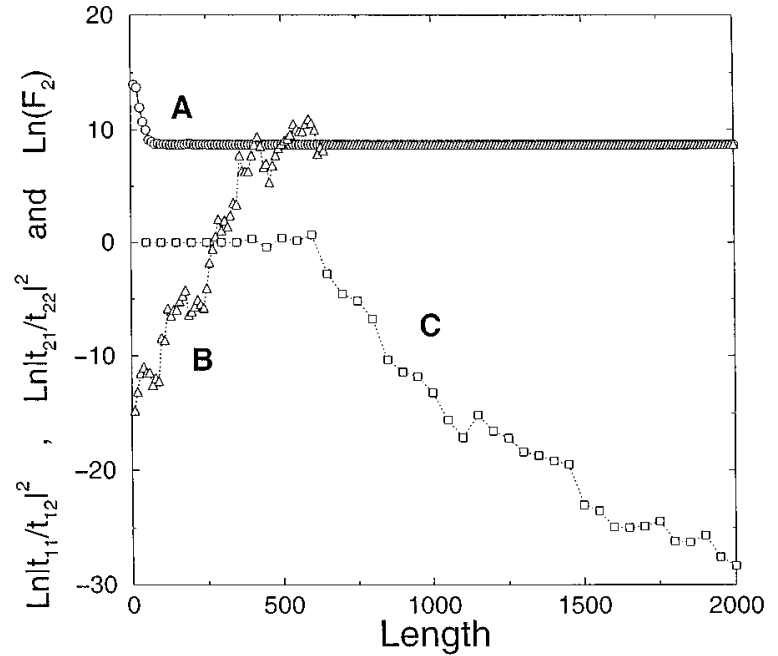

FIG. 1. At $W=1, W_{b}=1000$, with channel energies $E_{1}=$ -0.002 and $E_{2}=1.598$, the logarithms of $\left|t_{11} / t_{12}\right|^{2}$ (curve A) and $\left|t_{21} / t_{22}\right|^{2}$ (curve B) are plotted as functions of $L$. The logarithm of $F_{2}(L) \equiv\left|\operatorname{det}\left[t^{(2)}\right]\right| t_{11} t_{22} \mid$ is also plotted in curve C.

function of barrier height $W_{b}$, it is found that the ratio $\left|g_{1}(1) / g_{1}(2)\right|^{2}$ is proportional to $W_{b}^{2}$, independent of $W$. After configurational averaging, we plot in Fig. 2 the lengths $\xi_{\text {eq }}$ (in triangles), $1 / \lambda_{1}$ (in circles), and $1 /\left(\lambda_{2}-\lambda_{1}\right.$ ) (in squares) vs $\ln \left(W_{b}\right)$. This figure shows a logarithmic dependence of $\xi_{\text {eq }}$ on $W_{b}$ for large $W_{b}$ with a slope close to 99 . The lengths $1 / \lambda_{1}$ and $1 / \lambda_{2}$ depend weakly on $W_{b}$ and decrease monotonically with the limiting values $1 / \lambda_{1} \cong 105$, and $1 / \lambda_{2} \cong 33$. These results show that the channel occupation ratios and the equilibration length are determined mainly by the evanescent waves, whereas all the Lyapunov experiments are not much affected. As a function of $W$, we again find that all the lengths $\xi_{\text {eq }}, 1 / \lambda_{1}$, and $1 / \lambda_{2}$ are proportional to $1 / W^{2}$ at small $W$. Similar results are found from calculations for a system of six layers with three propagating channels and three alternate barriers. In order to understand these behaviors, we propose the following rate equation approach to this problem.

First, we rewrite the Hamiltonian of Eqs. (1) and (2) in the eigen channel representation, i.e., in terms of $E_{n}$ and $\varphi_{n}$,

$$
H=H_{0}+H_{1},
$$

with

$$
H_{0}=\sum_{n, i} E_{n} a_{n, x_{i}}^{\dagger} a_{n, x_{i}}+\sum_{n,[i, j]} a_{n, x_{i}}^{\dagger} a_{n, x_{j}}
$$

and

$$
H_{1}=\sum_{n, i} S_{n, n}\left(x_{i}\right) a_{n, x_{i}}^{\dagger} a_{n, x_{i}}+\sum_{n \neq m, i} S_{n, m}\left(x_{i}\right) a_{n, x_{i}}^{\dagger} a_{m, x_{i}},
$$

where

$$
S_{n, m}\left(x_{i}\right)=\sum_{z} \delta_{z, x_{i}} \varphi_{n}^{*}(z) \varphi_{m}(z)
$$

Here, $i$ is the site index along the $x$ axis and $[i, j]$ denotes the nearest-neighbor sites $i$ and $j$ in the same lateral plane. $H_{0}$ gives the propagation of each channel $n$ in the lateral direction. The function $S_{n, n}\left(x_{i}\right)$ in the first term of $H_{1}$ describes the disorder in the lateral plane for channel $n$ and, therefore, is responsible for the localization of that channel. The function $S_{n, m}\left(x_{i}\right)$ in the second term of $H_{1}$ provides the hoppings between channels $n$ and $m$. Both the localization and hopping mechanisms arise from the isotropic randomness $\delta_{z, x}$, or the term $\delta_{\alpha}$ in Eq. (2).

In the anisotropic regime, the overlap of wave functions between any two channels is small. All the important wave interference effects are due to the multiple scatterings of $S_{n, n}\left(x_{i}\right)$. These effects can be described by the localization length of each channel $\xi_{n}$. Since the cross-channel scattering terms $S_{n, m}\left(x_{i}\right)$ are weak, channel hoppings can be treated incoherently. Therefore, we can describe the lateral transport by a classical rate equation,

$$
\frac{d P_{n}}{d L}=\sum_{m \neq n}^{N}\left(w_{n m} P_{m}-w_{m n} P_{n}\right)-\frac{2 P_{n}}{\xi_{n}},
$$

where $P_{n}(L)$ is the outgoing wave probability in the propagating channel $n$ and $w_{n m}$ is the channel hopping rate, describing the probability of hopping from channel $m$ to $n$ per unit sample length. From the Born approximation, the hopping rate can be written as

$$
w_{n m}=\frac{\pi W^{2}}{6 \nu_{m}} \rho_{1 \mathrm{D}}\left(E-E_{n}\right) \sum_{z}\left|\varphi_{n}(z) \varphi_{m}(z)\right|^{2},
$$

where we have set $\hbar=1 . \nu_{m}$ is the wave velocity of channel $m$, i.e., $\nu_{m}=2 \sin k_{m}$, and $\rho_{1 \mathrm{D}}$ is the 1D density of state per site. Equation (14) is valid only if the channel hopping rate $w_{n m}$ is smaller than the channel decay rate $2 / \xi_{n}$ so that the separation between the effects due to localization and hoppings is possible. This is true in the anisotropic regime, $W_{1}$ $\gg 1 / W_{b}$, where channels are strongly localized and $w_{n m}\left[\propto(W / W b)^{2}\right] \ll 2 / \xi_{n}\left(\propto W^{2}\right)$.

The eigenvalues $\gamma i$ of the matrix

$$
q_{n m}=w_{n m}-\left(\sum_{\ell \neq n} w_{\ell n}+2 / \xi_{n}\right) \delta_{n m}
$$

on the right-hand side of Eq. (14) correspond to twice the Lyapunov exponents, i.e., $\gamma_{i}=2 \lambda_{i}$. The eigenvector $v_{1}(n)$, corresponding to the longest decay length $1 / \gamma_{1}$, determines the asymptotic channel occupation ratios, i.e., $P_{n} / P_{m}$ $=v_{1}(n) / v_{1}(m)$, which are independent of initial condition. Given an initial condition, say, $P_{n}(L=0)=\delta_{n m}$, the solution of $P_{n}(L)$ corresponds to $\left|t_{n m}(L)\right|^{2}$. The equilibration length $\zeta_{\text {eq }}$ can be determined from the condition that $P_{n}\left(\zeta_{\text {eq }}\right) / P_{m}\left(\zeta_{\text {eq }}\right) \cong v_{1}(n) / v_{1}(m)$ for all $n$ and $m$.

Let us apply the rate equation to the four channel case discussed above. Explicit expressions for the hopping rates $w_{12}$ and $w_{21}$ can be obtained from a perturbation calculation of channel eigenfunctions. We find from Eq. (15) $w_{12}$ $=a_{12}\left(W / W_{b}\right)^{2}$ and $w_{21}=a_{21}\left(W / W_{b}\right)^{2}$, where $a_{12}$ and $a_{21}$ are constants, independent of $W$ and $W_{b}$. By solving the eigenvalue problem for the matrix $q_{n m}$ of the rate equation, the decay exponents found are $\gamma_{i} \cong 2 / \xi_{i}+0.5\left(a_{12}\right.$ 


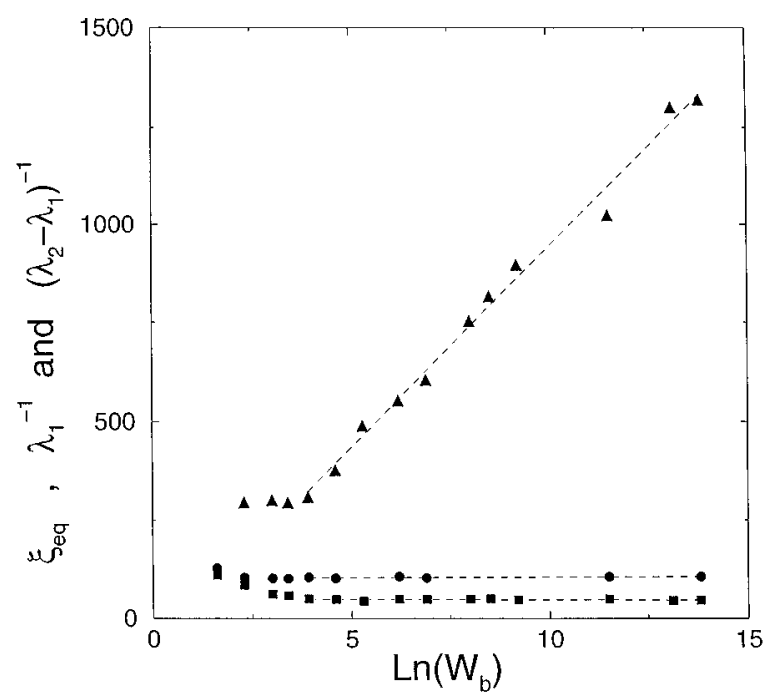

FIG. 2. At $W=1$ and channel energies $E_{1}=-0.002$ and $E_{2}=1.598$, the lengths $\xi_{\text {eq }}$ (in triangles), $1 / \lambda_{1}$ (in circles), and $1 /\left(\lambda_{2}-\lambda_{1}\right)$ (in squares) are plotted as function of $\ln \left(W_{b}\right)$.

$\left.+a_{21}\right)\left(W / W_{b}\right)^{2}$ for $i=1$ and 2 . Thus, in the large $W_{b}$ limit, the Lyapunov exponents of the system are just the perturbations from the inverse localization length of each channel. With the channel energies $E_{1} \cong 0$ and $E_{2} \cong 1.6$, the 1D localization lengths for $W=1$ and $\xi_{1} \cong 104$ and $\xi_{2} \cong 33$, respectively. ${ }^{10}$ These values are in good agreement with the limiting values of $1 / \lambda_{1} \cong 105$ and $1 / \lambda_{2} \cong 33$ shown in Fig. 2 . From the eigenvector $v_{1}$, we find the channel occupation ratio $P_{1} / P_{2}=\left(W_{b} / W\right)^{2}\left(2 / \xi_{2}-2 / \xi_{1}\right) / a_{12}$. This result shows explicitly that the dominant channel is determined by the one with the longest channel localization length, which has the channel energy closest to zero. ${ }^{10}$ Since both $\xi_{1}$ and $\xi_{2}$ are inversely proportional to $W^{2}$ at small $W$, the ratio $P_{1} / P_{2}$ becomes proportional to $W_{b}^{2}$ and is independent of $W$. This is consistent with the behavior of $\left|g_{1}(1) / g_{1}(2)\right|^{2}$ found numerically. Finally, the equilibration length obtained has the expression $\zeta_{\mathrm{eq}} \cong 2\left(\ln W_{b}\right) /\left(1 / \xi_{2}-1 / \xi_{1}\right)$, or $\zeta_{\mathrm{eq}} \propto\left(\ln W_{b}\right) / W^{2}$. At $W=1$, using the value of $\xi_{1} \cong 104$ and $\xi_{2} \cong 33$, the slope of $\zeta_{\text {eq }} / \ln W_{b}$ has the value 97 . This again agrees with the slope shown in Fig. 2. Thus, all the lateral transport behaviors obtained numerically are well reproduced by the rate equation. For the case of six channels with three alternate barriers, the results obtained from the rate equation also agree with those of recursive Green's function calculations. Since
1D channel localization length $\xi_{n}$ and density of states $\rho_{1 \mathrm{D}}$ are easily obtained, ${ }^{10}$ the rate equation approach provides a much simplified method for the study of lateral wave transport in the anisotropic regime.

If we assume a constant $W_{b}$ for all the barriers, the system considered here recovers the anisotropic hopping model ${ }^{3}$ in the limit $W_{1}=0$. In this limit, since channel wave functions are extended and overlap completely among themselves, the off-diagonal terms $S_{n, m}$ become the same order as the diagonal terms $S_{n, n}$ in Eq. (12). Strong multiple scatterings among channels make the channel-hopping descriptions inappropriate. Since both $w_{n m}$ and $2 / \xi_{n}$ are now proportional to $W^{2}$, Eq. (14) becomes invalid. However, the ergodicity in channel space dictates a transport behavior which belongs to the same universality class as an isotropic system. ${ }^{2}$ When $W_{1} \gg 1 / W_{b}$, channels are strongly localized and mixing of channels is weak and nonergodic. Only in this limit, is the channel-hopping mechanism an appropriate description of the lateral transport. It also facilitates the wave transport along the layering direction. The fact that the equilibration length becomes much longer than all channel localization lengths causes the concentration of probability on the channel with the largest localization length and, therefore, dictates a 1D-like behavior. Even in the weakly localized regime, i.e., $W_{1} \ll 1 / W_{b}$, where the width of channel eigenfunctions $\xi_{1 \mathrm{D}}$ are large in size, i.e., $\xi_{1 \mathrm{D}} \propto 1 /\left(W_{1}\right)^{2}$, if we divide the $z$ axis into blocks of size $\xi_{1 \mathrm{D}}$, the mixing of channels within each block is strongly due to large overlaps of channel wave functions. The effective localization length of each block is increased due to a reduction in the effective randomness from $W^{2}$ to $W^{2} / \xi_{1 \mathrm{D}}$. However, the mixing of channels between two spatially separated blocks could be weak. In an infinite sample, one can always find two wellseparated blocks so that the channel hopping rate between them is smaller than the effective decay rate of each block. Therefore, the equilibration length could become longer than all effective block localization lengths. This would lead to a concentration of probability near the block with the largest block localization length. This also implies that the anisotropic hopping model is unstable against a small perturbation of layer randomness, and, as a result, may lead to a 1D-like behavior; as is strongly indicated in the previous 3D study of Eqs. (1) and (2). ${ }^{1}$

The authors thank P. Sheng, B. White, and B. Derrida for useful discussions, and W.-L. Chan for helpful computational assistance.
${ }^{1}$ W. Xue, P. Sheng, Q. J. Chu, and Z. Q. Zhang, Phys. Rev. Lett. 63, 2837 (1989); Z. Q. Zhang, Q. J. Chu, W. Xue, and P. Sheng, Phys. Rev. B 42, 4613 (1990).

${ }^{2}$ P. Sheng and Z. Q. Zhang, Phys. Rev. Lett. 74, 1343 (1995).

${ }^{3}$ W. Apel and T. M. Rice, J. Phys. C 16, L1151 (1983); Q. Li, C. M. Souloulis, E. N. Economou, and G. S. Grest, Phys. Rev. B 40, 2825 (1989).

${ }^{4}$ P. A. Lee and D. Fisher, Phys. Rev. Lett. 47, 882 (1981).

${ }^{5}$ A. D. Stone, P. A. Mello, K. A. Muttalib, and J. L. Pichard, in Mesocopic Phenomena in Solids, edited by B. L. Altshuler, P. A. Lee, and R. A. Webb (North-Holland, Amsterdam,
1991).

${ }^{6}$ For instance, see A. Crisanti, G. Paladin, and A. Vulpiani, Products of Random Matrices in Statistical Physics (Springer-Verlag, Berlin, 1993).

${ }^{7}$ I. Goldhirsch, P. L. Sulem, and S. A. Orszag, Physica 27D, 311 (1987).

${ }^{8}$ A. Mackinnon and B. Kramer, Z. Phys. B 53, 1 (1983).

${ }^{9}$ W. Kohler, G. Papanicolaou, and B. White, Wave Motion (to be published).

${ }^{10} \mathrm{P}$. Sheng, Introduction to Wave Scattering, Localization, and Mesoscopic Phenomena (Academic, New York, 1995). 